\title{
PRESUPUESTOS, SENTIDOS Y PRÁCTICAS AMBIENTALES EN LA CIÉNAGA DE MALLORQUÍN Y SUS ÁREAS DE INFLUENCIAS EN RELACIÓN CON EL DESARROLLO SOCIAL Y HUMANO PARA TRANSFORMAR LAS CONDICIONES DE VIDA DEL SECTOR, CIUDAD O REGIÓN*
}

Articulo de Reflexion - Recibido: 12 de Agosto de 2014 - Aceptado: 15 de Noviembre de 2014

Arq. Ruben Dario Franco Medina*

Corporación Universidad de la Costa - CUC

Para citar este artículo / to reference this article:

Franco, R. (2015). Presupuestos, sentidos y prácticas ambientales en la Ciénaga de Mallorquín y sus áreas de influencias en relación con el desarrollo social y humano para transformar las condiciones de vida del sector, ciudad o región. Módulo Arquitectura CUC, Vol.14 N² 84-104. Doi:

\section{Resumen}

En el presente artículo se reflexiona acerca de las problemáticas ambientales y humanas de la Ciénaga de Mallorquín, con el objetivo primordial de establecer nuevos sentidos y prácticas ambientales que satisfagan las necesidades humanas fundamentales de los habitantes de dicho humedal, generando niveles crecientes de autodependencia y articulación orgánica con la naturaleza y la tecnología, lo que permitirá solucionar muchos de los problemas ambientales y humanos vigentes en dicho ecosistema y su área de influencia.

Palabras Clave:

Ciénaga de Mallorquín, desarrollo sostenible, prácticas ambientales, región

Articulo producto de la investigación terminada de la Maestría en Desarrollo sostenible y medio ambiente de la Universidad de Manizales

" Arquitecto docente de la Corporación Universidad de la Costa- CUC, Magister en Desarrollo Sostenible y medio ambiente de la Universidad de Manizales. 


\section{BUDGETS, SENSES AND ENVIRONMENTAL PRACTICES SWAMP MALLORQUINN AND AREAS OF INFLUENCE IN CONNECTION WITH SOCIAL AND HUMAN DEVELOPMENT TO TRANSFORM THE LIVING CONDITIONS OF SECTOR , CITY OR REGION}

\section{Abstract:}

This paper reflects on the environmental and human problems of Majorcan Swamp, with the purpose of establishing new meanings, and practices that meet the basic human needs of the inhabitants of the wetland, generating increasing levels of self-reliance and organic articulation with nature and technology, which will solve many of the environmental and human problems existing in this ecosystem and its area of influence.

Key Words:

Ciénaga de Mallorquín, sustainable development, environmental practices 


\section{Introducción}

Hoy es aceptado casi como un escenario común, que el desarrollo y las necesidades humanas son componentes de una misma ecuación. Sin embargo, eso no siempre fue así. La evolución de un concepto tan importante como el de desarrollo sostenible , tuvo muchos obstáculos y enemigos cuyos intereses obedecen a diferentes modelos de desarrollo económicos imperantes en gran parte del planeta, y a quienes no les preocupa la degradación ambiental mundial, bien sea porque están inmersos en procesos de industrialización o bien porque, de algún modo u otro, les conviene mantener intacto el actual estado de cosas.

En efecto, a escala global, la contaminación ambiental se ha ido incrementando al igual que la inequidad, para lo cual, hoy no basta simplemente con proponer fórmulas tendientes a contrarrestar las problemáticas ambientales o a proteger la biodiversidad de determinado ecosistema, sino que se debe mirar íntegramente la idea de desarrollo, para que los humanos participen de las soluciones a los problemas que los afectan y se beneficien de las mismas junto con la naturaleza. Significa entonces reconocer la incompletitud e insuficiencia de las teorías económicas y sociales que han servido de sustento y orientación a los procesos de desarrollo hasta el presente.

\section{Evolución histórica del Desarrollo Sostenible, elementos esenciales y caracterizaciones teóricas}

En el año de 1972 se publicó un importante estudio Ilamado "Los límites del crecimiento" para el Club de Roma (respaldado por diversos científicos ambientales, políticos y profesionales) en el que, según el economista Alejandro Nadal (2007:14), se plantea por primera vez el concepto de desarrollo sustentable y se pone de presente la preocupación mundial en torno al incremento de la población, la industrialización, la contaminación y el agotamiento de los recursos naturales, por lo que, los límites al crecimiento serían alcanzados en algún momento durante los siguientes cien años, ya que se produciría un colapso en los niveles absolutos de población mundial y producción industrial.

Lo anterior se generó en el marco del creciente deterioro ambiental del planeta y en la necesidad de asumir, por medio de la cooperación internacional, la creación y cumplimiento de programas globales, nacionales y regionales que contrarrestaran los efectos del deterioro, para asegurar la protección del medio ambiente. De ahí que, posteriormente surgirían instancias de discusión sobre las estrategias de desarrollo sustentable y equitativo que deberían implementar los diversos países del mundo. El resultado de esto fue la Cumbre de Naciones Unidas sobre el Medio Humano (Cumbre 
de Estocolmo) en 1972, en el que se acordaron algunos principios sobre el medio ambiente y el desarrollo, y se recomendaron planes de acción ambiental a escala global. En 1984 se hizo evidente que sería mucho más preciso dar mayor impulso a las políticas ambientales y hacerlas parte de los planes de desarrollo. Fue así como la Asamblea General de las Naciones Unidas dio vida a una comisión Mundial del Medio Ambiente y el Desarrollo, presidida por la doctora Gro Harlem Brundtland (primera ministra de Noruega) e integrada por 24 personalidades de todas las regiones del mundo. La Comisión Brundtland, así llamada, instalada en Ginebra, dio a conocer al público su informe en 1987, después de múltiples consultas regionales con las comunidades científicas-ecológicas. Dicho informe constituyó una nueva base para dar consistencia conceptual a la labor de las Naciones Unidas en materia ambiental, ya que se propugnó por un desarrollo que respetara la conservación de la naturaleza, redujera las contaminaciones y los deterioros existentes. Se debía dar impulso además a procesos de producción limpios en la agricultura, la industria y los servicios, con las necesarias adaptaciones del transporte, el crecimiento urbano y el industrial para hacer viables los propósitos de un desarrollo que se llamaría sustentable y equitativo, como legado indispensable a las generaciones venideras para su supervivencia. Al respecto, el informe menciona textualmente lo siguiente:
"Está en manos de la humanidad hacer que el desarrolla sea sostenible, duradero, a sea, asegurar que satisfaga las necesidades del presente sin comprometer la capacidad de las futuras generaciones para satisfacer las propias. El concepto de desarrollo duradera implica límites -no límites absolutos, sino limitaciones que imponen a las recursos del medio ambiente el estado actual de la tecnología y de la organización social, la capacidad de la biósfera de absorber los efectos de las actividades humanas. Pero tanto la tecnología como la organización social pueden ser ordenadas y mejoradas de manera que abran el camino a una nueva era de crecimiento económico. La Comisión cree que ya no es inevitable la pobreza general. La pobreza es no es sólo un mal en sí misma. El desarrollo duradero exige que se satisfagan las necesidades básicas de todos y que se extienda a todos la oportunidad de colmar sus aspiraciones a una vida mejor. Un mundo donde la pobreza es endémica estará siempre propenso a ser víctima de la catástrofe ecológica o de otro tipo." (Brudtland, 1987)

Por lo demás, ha quedado claro que las especies amenazadas no son solo las pertenecientes a la fauna y la flora, sino la especie humana misma. Los efectos de la actividad económica sobre los recursos naturales son equiparables a los producidos en la salud humana por las contaminaciones, en diferentes lapsos según las especies y localizaciones territoriales. 
Posteriormente, como consecuencia de los debates en el Programa de las Naciones Unidas para el Desarrollo (PNUD) sobre el Desarrollo Humano, a principios de los años noventa, surgieron importantes definiciones de desarrollo sostenible como un concepto amplio e integral, que comprende todas las opciones humanas, en todas las sociedades y en todas las etapas de desarrollo, de esta manera:

"Expande el diálogo sobre el desarroIlo, pues éste deja de ser un debate en torno a los solos medios (crecimiento del producto nacional bruto, PNB) para convertirse en un debate sobre los fines últimos. Al desarrollo humano le interesan tanto la generación de crecimiento económico como su distribución, tanto las necesidades básicas como el espectro total de las aspiraciones humanas, tanto las aflicciones humanas del norte como las privaciones humanas del sur. El concepto de desarrollo humano no comienza a partir de un modelo predeterminado. Se inspira en las metas de largo plazo de una sociedad. Teje el desarrollo en torno a las personas, y no las personas en torno al desarrollo". (Programa de la Naciones Unidas para el desarrollo, 1992)

Y en otro momento señala que este proceso de desarrollo debe por lo menos propiciar un ambiente adecuado para que todas las personas puedan desarrollar todo su potencial y tener oportunidades razonables para llevar una vida productiva, creativa y que satisfaga todas sus necesidades e intereses. (Programa de la Naciones Unidas para el desarrollo, 1992).

Luego de esto, la Conferencia de las Naciones Unidas sobre el Medio Ambiente y el Desarrollo, celebrada en 1992 la Cumbre de la Tierra de Río de Janeiro (Cumbre de Río), adoptó un programa de acción para el siglo XXI (Programa 21) que busca proteger la biodiversidad, controlar la deforestación, proteger los bosques en general y evitar la constante pérdida de suelos. La Declaración de Río contiene 27 principios, entre los que se destacan los siguientes:

- Los seres humanos tienen derecho a una vida saludable y productiva en armonía con la naturaleza.

- El derecho al desarrollo debe ejercerse en forma tal que responda equitativamente a las necesidades ambientales y de desarrollo de las generaciones actuales y futuras.

- Erradicar la pobreza y reducir las disparidades en los niveles de vida en los distintos pueblos del mundo es indispensable para el desarrollo sostenible.

- La protección del medio ambiente constituye parte integrante del proceso de desarrollo y no puede considerarse en forma aislada. 
- Las medidas internacionales que se adopten con respecto al medio ambiente y el desarrollo deben considerar también los intereses y necesidades de todos los países.

- Para alcanzar el desarrollo sostenible y una mejor calidad de vida para todas las personas, los Estados deberán reducir y eliminar las modalidades de producción y consumo insostenibles y fomentar políticas demográficas apropiadas.

- Las mujeres desempeñan un papel fundamental en la gestión ambiental y el desarrollo. Por lo tanto, es imprescindible contar con su plena participación para

- Lograr el desarrollo sostenible.

- La guerra es intrínsecamente destructiva para el desarrollo sostenible. La paz, el desarrollo y la protección del medio ambiente son interdependientes e inseparables.

Según la Organización de las Naciones Unidas para la Educación, la Ciencia y la Cultura (UNESCO, 2012) estos principios pueden guiar las acciones de los gobiernos, las comunidades y las organizaciones para definir los objetivos de sostenibilidad y crear programas para ayudar a lograr estos objetivos. El Informe sobre Desarrollo Humano del PNUD correspondiente al año 2000 precisa mejor estas ideas y señala que el desarrollo humano incluye, además de algunos indicadores económicos y sociales, "otras esferas de opciones", como "la participación, la seguridad, la sostenibilidad, las garantías de los derechos humanos, todas necesarias para ser creativo y productivo y para gozar de respeto por sí mismo, potenciación y una sensación de pertenecer a una comunidad. En definitiva, el desarrollo humano es el desarrollo de la gente, para la gente y por la gente". $Y$ el mismo texto enfatiza: "Los derechos humanos y el desarrollo humano tienen una visión común y un propósito común: velar por la libertad, el bienestar y la dignidad de todos en todas partes. (Programa de la Naciones Unidas para el desarrollo, 1992) Pero, este avance tiene un sustento esencial en la Declaración Universal de los Derechos Humanos, aprobada por la Asamblea General de las Naciones Unidas en diciembre de 1948, cuyo artículo 25 preceptúa que "Toda persona tiene derecho a un nivel de vida adecuado que le asegure, así como a su familia, la salud y el bienestar, y en especial la alimentación, el vestido, la vivienda, la asistencia médica y los servicios sociales necesarios; tiene asimismo derecho a los seguros en caso de desempleo, invalidez, viudez, vejez u otros casos de pérdida de sus medios de subsistencia por circunstancias independientes de su voluntad (19)". Lo que proponen las diversas cumbres e informes, mencionados anteriormente, sobre el desarrollo sostenible y humano, puede ser representado de la siguiente manera: 


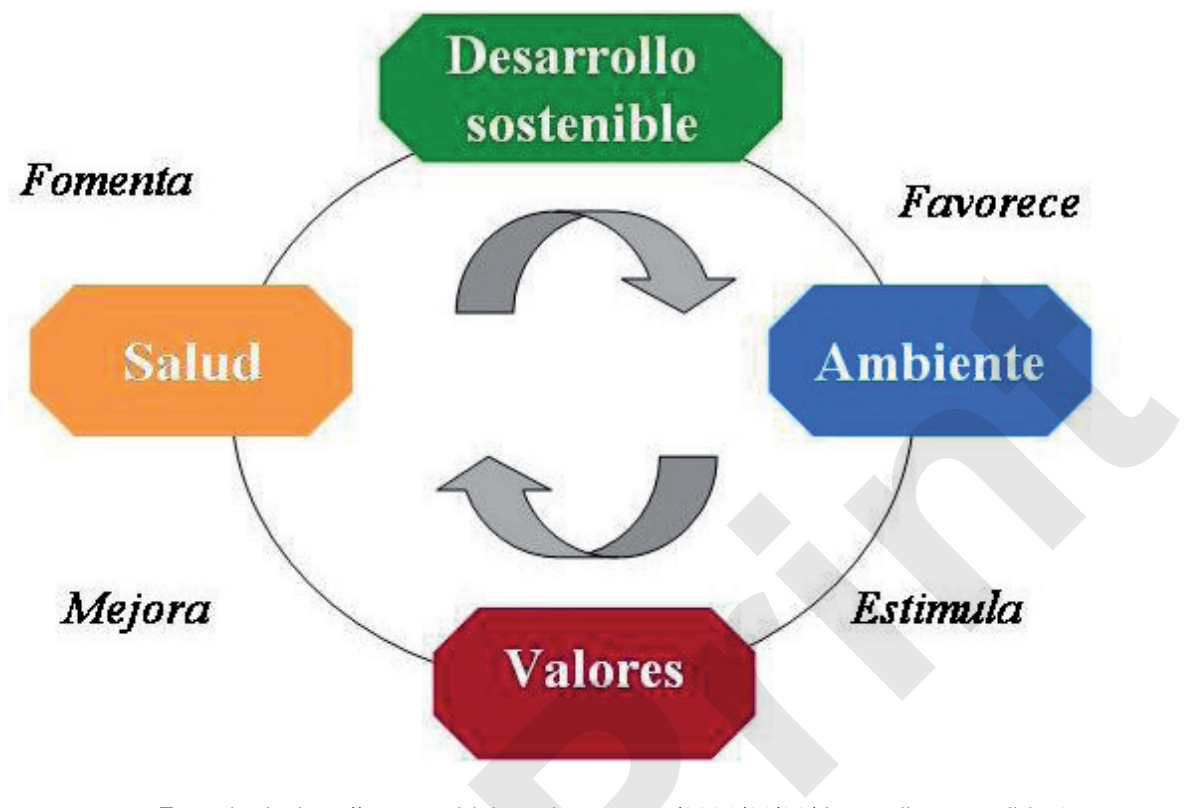

Tomado de: http://carmenvidal.wordpress.com/2008/01/22/desarrollo-sostenible-2

De esta manera, queda claro que la noción de desarrollo sostenible cuenta con diversos elementos, esenciales para satisfacer los niveles básicos de los seres humanos, dentro de los que, según la profesora Gemma Cervantes (2005:76), cabe destacar:

- Solidaridad Intrageneracional: El concepto habla de satisfacer las necesidades actuales $\mathrm{y}$, por tanto, habla implícitamente de solidaridad intrageneracional. No debe tener ningún sentido pensar que sólo nos preocupen nuestras necesidades. En un planeta tan superpoblado como el nuestro y con unos recursos escasos y que decrecen con rapidez, si una parte de la población consume grandes cantidades de recursos, el resto no tendrá el mínimo necesario. Por tanto, la distribución equitativa (solidaridad Intrageneracional) se convierte en un requisito previo para cumplir el objetivo básico de preservar y perdurar la vida (solidaridad Intrageneracional).

- Solidaridad Intrageneracional: El concepto habla también de no comprometer la capacidad de las generaciones futuras de satisfacer sus propias necesidades, vuelve p o r tanto a hablar de necesidades, ahora en una perspectiva de futuro y habla por tanto de solidaridad Intrageneracional. 
- Necesidades: El concepto habla básicamente de necesidades, por tanto, será necesario profundizar cuales y de quienes son las necesidades a satisfacer para poder definir estrategias y tácticas para caminar hacia la sostenibilidad.

- En ese sentido, las capacidades básicas para el desarrollo humano se dirigirán, en todos los niveles, a:

- Obtener una vida más larga y saludable

- Conseguir conocimientos

- Acceder a los recursos necesarios para un nivel de vida adecuado

En definitiva, cuando hablamos de desarrollo sostenible, debemos entender lo siguiente (Nadal, 2007):

1. El desarrollo sustentable y equitativo es una estrategia, en los ámbitos global, regional y nacional, que permite entregar en forma progresiva a las generaciones venideras la posibilidad de mejorar su calidad de vida sin destrucción de los recursos naturales renovables del planeta y con protección de la biodiversidad.

2. El desarrollo sustentable deberá proseguirse en condiciones de creciente eficiencia tanto económica como ambiental en el uso de los recursos naturales y en la producción de bienes y servicios. El saber científico y tecnológico deberá emplearse para proteger los recursos tanto renovables como los no renovables, con transiciones hacia métodos de producción que descansen en el empleo de insumos materiales menos contaminantes de los suelos, las aguas, el atmósfera y el hábitat rural y urbano de la especie humana.

3. El desarrollo sustentable habrá de ser equitativo, es decir deberá comprender la idea tradicionalmente aceptada de contribuir mediante políticas y programas económicos y sociales articulados, a reducir las gruesas desigualdades que se han manifestado durante el siglo XX, que hoy alcanzan extremos que están en evidencia en todos los continentes mediante diversos indicadores que están en esta era de la comunicación instantánea, no pueden ni deben ser ocultados a la conciencia ciudadana. El desempleo y la miseria no tienen cabida en un desarrollo sustentable y equitativo.

4. No se trata, en consecuencia de hacer más de lo mismo que ha presencia ya el siglo $X X$, sino de emprender un proceso que, además de considerar la inversión real productiva para lograr índices de bienestar económico más elevados, tenga en cuenta de manera simultánea la inversión ambiental requerida y lo que ahora se llama eco efi- 
ciencia, así como la necesidad de construir el bienestar social sobre bases equitativas. La formación de los recursos humanos vía educación y la capacitación deberá constituir uno de los pilares del desarrollo sustentable y equitativo.

Visto esto, podemos decir en referencia a la recuperación del ecosistema de la Ciénaga de Mallorquín (objeto principal de esta investigación), que para mejorar la calidad de vida de sus habitantes, se debe empezar por establecer estrategias de corto, mediano y largo plazo que propugnen por planes sostenibles con el medio ambiente. Las problemáticas de la Ciénaga no han alcanzado la prioridad necesaria que se merece en su condición de humedal, ni en los gobiernos locales, ni en los nacionales y mucho menos en la sociedad civil. Así que, se debe empezar por aplicar la noción de desarrollo sostenible, con todas sus implicaciones, en esta zona del país, para que no solo se atiendan las problemáticas que alteran el equilibrio de ese ecosistema de la humanidad, sino también para crear condiciones materiales favorables a los habitantes marginados de ese sector. De esa manera, se estaría respondiendo a las problemáticas de la Ciénaga de manera integral, es decir, adoptando medidas para la protección del humedal pero también generando desarrollo humano y equitativo a lo largo de ese territorio.

\section{Crítica al modelo de desarrollo imperante en el país y América Latina}

A pesar de todos los esfuerzos mencionados en el punto anterior para implementar, a nivel mundial, modelos de desarrollo acordes con la naturaleza, el Programa de las Naciones Unidas para el Medio Ambiente (PNUMA) realizó en 1997 una exhaustiva evaluación sobre las "Perspectivas del Ambiente Mundial" (GEO-1, por sus siglas en inglés), en el que se obtienen resultados muy negativos en materia de sostenibilidad ambiental. Es decir, que "a pesar de los avances en varios frentes, el ambiente, desde una perspectiva global, ha seguido degradándose durante el último decenio, y problemas ambientales destacados siguen estando enraizados en el tejido socioeconómico de las naciones de todas las regiones". Más adelante, se recalca en dicho informe que:

Según United Nations Environment Programme (UNEP-PNUMA, 1997): "el progreso hacia un futuro global es demasiado lento. No se aprecia ningún sentido de urgencia. Tanto en el ámbito internacional como en el nacional son todavía insuficientes los recursos financieros disponibles y la voluntad política para frenar la degradación ambiental global y para hacer frente a los problemas más importantes -por más que se disponga ya del conocimiento y la tecnología necesaria para lograrlo. Se reconoce en forma apenas limitada que los problemas 
ambientales son por fuerza de largo plazo y acumulativos, y que tienen graves consecuencias globales y en materia de seguridad (...) Las estructuras para la gobernación y solidaridad ambiental globales siguen siendo demasiado débiles para que el progreso se alcance como realidad mundial. Como resultado de todo ello, se está ampliando la brecha entre lo que se ha conseguido y lo que con sentido realista falta por hacer.

En el informe GEO 2000, publicado en junio del 2000 por el mismo organismo, se advierte de la necesidad de investigar las causas de los principales problemas ambientales. En cuanto a los esfuerzos que se han hecho para detener el deterioro ambiental, el informe expresa:

Son pocos y tardíos. Al ambiente se asigna todavía baja prioridad en la planeación nacional y regional, y son escasos los recursos financieros si se comparan con los que se asignan a otras áreas del desarrollo. Pese a señales positivas y a mejoramiento de las políticas ambientales, faltan procesos de formulación integrales -cada vez más urgentes- que deben reflejarse, además, en las evaluaciones (UNEP- PNUMA,1999).

En la región Latinoamericana y del Caribe, a pesar de haber sido la sede de la Cumbre de Río en 1992 y de haberse obtenido respuesta positiva de las sociedades civiles de muchos de los países zaciones locales, y de haberse llevado a la práctica diversos programas ambientales, el avance pro ambiental, según lo explica el profesor Nadal (2007: 70) ha sido mínimo y, por otro lado, el deterioro ha sido constante y de gran peligro para la especie humana, y no sólo para los equilibrios ecológicos.

Un informe de la Comisión Económica para América Latina y el Caribe (CEPALC) en el 2001, reconoce el desafío que presentan las tendencias de deterioro ambiental como parte del proceso de desarrollo, es decir, empieza a considerar seriamente la noción de desarrollo sustentable en la región. A partir la aseveración de que "la creciente conciencia internacional respecto de los aspectos ambientales del desarrollo ha penetrado gradualmente en las políticas públicas de la región", se afirma en seguida "que a pesar de los esfuerzos realizados, la información sobre el estado del medio ambiente muestra que el proceso de degradación ha seguido avanzando en los últimos años", lo que compromete el desarrollo futuro de los países de la región, cuyas economías dependen en gran medida del mantenimiento en el largo plazo de la capacidad productiva de los ecosistemas".

Por su parte el PNUMA- ORPALC (Oficina Regional para América Latina y el Caribe), en un informe sobre América Latina y el Caribe, afirma: "Los albores del siglo XXI, nos presentan un mundo 
en donde continúan la contaminación, la degradación y la destrucción del medio ambiente. $Y$ añade de inmediato: "El tiempo en el que el medio ambiente era considerado tema adicional, ha pasado. Ahora es fundamental, que el tema ambiental sea definitivamente integrado en el proceso de toma de decisiones en todos los sectores, tales como la agricultura, la economía y la gestión urbana" (PNUMA, 2000).

La CEPALC cita información del banco mundial y el fondo mundial para la naturaleza (WWF, por sus siglas en inglés) según la cual "un 46\% de las ecoregiones terrestres de América Latina y el Caribe se encuentra en estado crítico, o en peligro, y un $31 \%$ en estado de vulnerabilidad -no solo bosques tropicales húmedos y de coníferas, sino además pastizales, matorrales y manglares- y considera que la deforestación continua siendo el principal problema ambiental, como proceso que es la causa principal de pérdida de biodiversidad (2001: 250-251)." Al decir de Alejandro Nadal (2007:75) "la región padece un fenomenal vertido de desechos a la intemperie y en superficies hídricas, sin tratamiento y sin procesos de confinamiento adecuados". Igualmente afirma que "la Organización Panamericana de la Salud (OPS), (estimó) que en 1995 se generaron 275.000 toneladas diarias de residuos sólidos urbanos (municipales)". Resulta además desolador para este autor mexicano que "menos del $2 \%$ de las aguas residuales de la región recibe tratamiento y que $40 \%$ de los desechos se lanza a tiraderos no controlados".

La persistencia de todas estas condiciones, y otras como la contaminación atmosférica, la pérdida constante de biodiversidad, la contaminación de las zonas costeras, la degradación de los suelos, indican que las políticas ambientales distan mucho de ser adecuadas. Si, como sostiene Baumol (2004) las economías capitalistas, imperantes en el mundo entero, son tan exitosas para producir crecimiento, deberíamos preguntar si tendrán el mismo éxito en proporcionar desarrollo sustentable. Es decir, se necesita saber si se puede transitar a economías que puedan incrementan el bienestar humano sin intensificar el uso de recursos naturales y energía que se encuentra en su entorno. "Esas economías no estarían estáticas, por el contrario, permanecerían en constante regeneración, pero tendrían que permanecer en una escala que permitiera al medio ambiente continuar sus funciones regulatorias de los grandes ciclos biológicos, geológicos y químicos del planeta".

Precisamente, en las últimas décadas se han generado análisis y críticas muy relevantes de diversos autores latinoamericanos a los modelos de desarrollo dominantes en la región. Esto nos permite comprender las actuales crisis no sólo ambientales sino sociales. Entre 
esos trabajos, podemos encontrar las agudas reflexiones realizadas por el economista chileno Manfred Max-Neef en colaboración con Antonio Elizalde y Martín Hopenhay (1998: 25-26), quienes consideran que en América Latina se disputan dos grandes concepciones económicas con plena vigencia: "el desarrollismo" y "el monetarismo neoliberal". Ambas tienen repercusiones graves sobre el medio ambiente, puesto que la primera de ellas (el desarrollismo) fracasó por su propia incapacidad para controlar los desequilibrios monetarios y financieros, ya que la estructura productiva que generó -especialmente la industria-resultó tremendamente concentradora, y a que su enfoque del desarrollo, predominantemente económico, descuidó otros procesos sociales y políticos que comenzaban a emerger con fuerza y gravitación crecientes, especialmente después del triunfo de la revolución cubana. La segunda por su parte (neo-liberalismo monetarista) es otra y bien distinta. A diferencia del desarrollismo, el neo-liberalismo monetarista ha fracasado en un período mucho más breve y de manera mucho más estrepitosa. Más aún, se parece a un derrumbe fenicio, que nada deja después de su paso excepto un inmenso vacío. Esto es así, según los sustentan estos autores al menos por tres razones:

Primero, porque a pesar de poder impulsar el crecimiento económico, no es generador de desarrollo en el sentido amplio que hoy lo entendemos.

Segundo, porque sus supuestos de racionalidad económica son profundamente mecanicistas e inadaptables, por lo tanto, a las condiciones de países pobres, donde la miseria no puede erradicarse como consecuencia de la liberalización de un mercado del que los pobres se encuentran, de hecho, marginados.

Tercero, porque en mercados restringidos y oligopólicos, donde los grupos de poder económico no se enfrentan a fuerzas capaces de limitar su comportamiento, la actividad económica se orienta con sentido especulativo, lo que deriva en resultados concentradores que son socialmente inaguantables.

Por último, se destaca que ambas concepciones económicas han compartido algunos elementos, aunque con distinta intensidad. Ambas suponen que la concentración estimula el crecimiento lo cual es demostrable estadísticamente -pero, mientras el neoliberalismo no ve necesidad alguna delimitarla, el desarrollismo, que sí le reconoce límites, no logra controlarla. De lo dicho se desprende que

Nuestra situación dista mucho de ser coyuntural. De allí que resulta inevitable, en nuestra opinión, desplegar todos los esfuerzos posibles para diseñar alternativas imaginativas pero viables. Las 
condiciones de tal -o de tales- alternativas parecen bastante claras. Por una parte, si las dos concepciones económicas que han dominado el escenario latinoamericano no han logrado satisfacer las legítimas carencias de las mayorías latinoamericanas, una nueva concepción ha de orientarse primordialmente hacia la adecuada satisfacción de las necesidades humanas. Por otra parte, si el desarrollo futuro no podrá sustentarse en la expansión de las exportaciones (por las barreras descritas), ni en sustanciales aportes de capital foráneo por las dramáticas limitaciones que impone la deuda externa, la nueva concepción ha de orientarse inevitablemente hacia la generación de una creciente autodependencia.

De igual manera, el científico social Theotonio Dos santos, en su libro "Imperialismo y dependencia" (2011), ofrece una perspectiva aguda y crítica como la anterior, en torno a los modelos de desarrollo vigentes en América Latina. Al respecto afirma:

La teoría del desarrollo que ha predominado en nuestros países ha puesto el énfasis en el tránsito de una sociedad atrasada, tradicional o feudal, etc., hacia una sociedad moderna, desarrollada o capitalista, etc. Este énfasis suponía que los problemas por resolver provienen del polo atrasado de estas economías e hizo que se concentrara el análisis científico en los obstáculos al desarrollo que se encontraban en estos polos atrasados.
En función de esta actitud metodológica básica, se ha elaborado un modelo de desarrollo de América Latina que confiaba fundamentalmente en los efectos económicos, sociales, políticos e ideológicos progresivos de la industrialización. Sin embargo, el transcurso de la industrialización en nuestros países no solo no ha eliminado gran parte de los obstáculos atribuidos a la sociedad tradicional, sino que ha creado nuevos problemas y tensiones muy agudas que se reflejan en una crisis general de América Latina (354).

De esta manera, este profesor brasileño al referirse a los "modelos" de desarrollo existentes en la región, plantea que no se pueden repetir y tampoco los "modelos" de sociedad desarrollada, ya que son cristalizaciones de metas por alcanzar. En el caso colombiano, el reconocido economista Sarmiento (1989) explica que "el país se enfrenta a un modelo de desarrollo que no da crecimiento ni equidad. $\mathrm{Ni}$ siquiera resuelve el problema del empleo dentro de un ambiente de altura demográfica". Para agravar más las cosas, "la apertura comercial indujo una especialización en actividades de baja productividad que enfrentan limitaciones en los mercados internacionales, como la minería, la agricultura tropical y la industria tradicional, y propició la sustitución de empleo formal por importaciones. La economía avanzó lentamente porque careció de la demanda que la impulsara y de la capacidad instalada que la sostuviera". 
Para Sarmiento (2010), la explicación de lo anterior "se encuentra en la invalidez de las concepciones clásicas y neoclásicas que sirvieron de fundamento al consenso de Washington, y la solución en sustituirlas por otras teorías más representativas de la realidad, por lo tanto, propone modificar la organización monetaria y financiera y construir una estructura industrial, que permitan crear las condiciones de ahorro y capitalización y de mercado interno y externo para sostener tasas de crecimiento superiores". Aún más importante, "es necesario conectar el área económica con la social para sustituir el asistencialismo por el empleo formal con mayor salario mínimo, seguridad de salud y pensiones y acceso a la educación de calidad".

Visto lo anterior, el término "crecimiento", desde la perspectiva económica del profesor Julio Silva (2007), debe entenderse como la creciente disponibilidad de bienes y servicios para satisfacer las necesidades materiales, sociales y espirituales de los seres humanos. $Y$ «desarrollo», también desde esa visión, debe verse como el mejoramiento de las condiciones de vida de los seres humanos por medio de la satisfacción de sus necesidades materiales, sociales y espirituales. Es decir, el "crecimiento" permite la disponibilidad de los bienes y servi- cios necesarios, lo que corresponde al campo de la oferta, de la producción, mientras el «desarrollo» es la utilización de esos bienes y servicios para vivir mejor, lo que corresponde más al campo de la demanda, sobre todo del consumo final. O sea, el «crecimiento» es el medio y el «desarrollo» es el fin.

La sostenibilidad, en consecuencia, es un paradigma para pensar en un futuro en el cual las consideraciones ambientales, sociales y económicas se equilibran en la búsqueda del desarrollo y de una mejor calidad de vida. Estos tres ámbitos -la sociedad, el medio ambiente y la economíaestán entrelazados. En efecto, los programas para el desarrollo sostenible deben considerar los tres ámbitos de la sostenibilidad -medio ambiente, sociedad y economía- así como también una dimensión subyacente de la cultura. Puesto que, el desarrollo sostenible se adecúa a los contextos locales de estos tres ámbitos, por lo que se adoptarán formas muy variadas en todo el mundo. En ese sentido, el desarrollo sostenible genera una interacción entre diversos campos sociales, que tejen una visión de desarrollo humano con los elementos propios de cada contexto. Veamos los siguientes diagramas de Mann (2006), que permiten comprender de mejor manera lo explicado con anterioridad: 


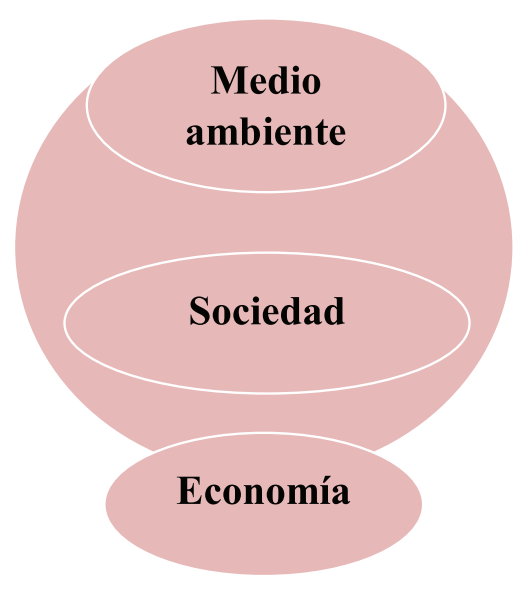

De esta manera, decimos que los ideales y principios que constituyen la sostenibilidad, como desarrollo humano alternativo, incluyen conceptos amplios tales como equidad entre las generaciones, equidad de género, paz, tolerancia, reducción de la pobreza, preservación, restauración e integración del medio ambiente, así como vitalidad económica, conservación de los recursos naturales y justicia social. En el tema que nos compete, la Ciénaga de Mallorquín lleva implícito el costo por la pérdida o deterioro de su biodiversidad. Su daño es difícil de medir, pero es significativo, y además está aumentando. Además, algunos de estos costos están siendo soportados de manera desigual por distintos grupos sociales, debido al modelo de desarrollo inequitativo que opera en el país. Todos estos problemas llevan a plantearse la necesidad de definir algún tipo de objetivos y criterios tecnoló-

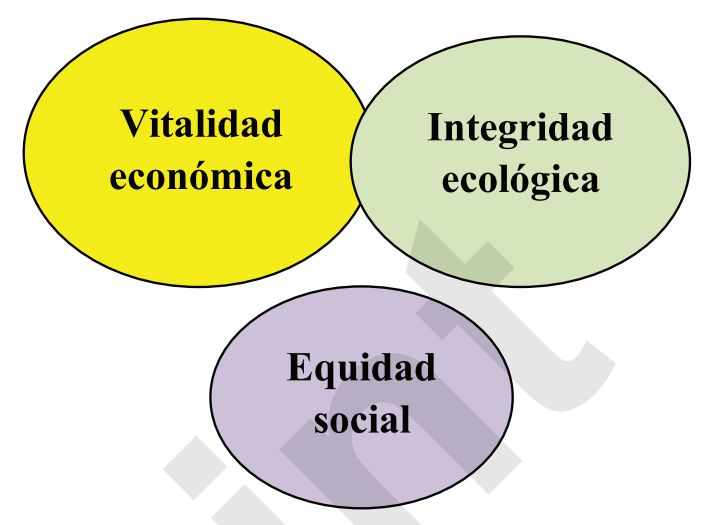

gicos para el desarrollo sostenible de ese humedal.

Resulta necesario entonces que los patrones de actividad humana en la Ciénaga se encuentren dentro de la capacidad de carga del medio (sostenibilidad ambiental) y de la sociedad (sostenibilidad social). Para ello, la ciudadanía debe redefinir completamente estos fines, patrones y maneras de consumo, reduciendo notablemente los niveles de actividad en dicho humedal. Por tal razón, es indispensable que en el país se propongan modelos propios de desarrollo, acompañando el crecimiento de la población con la capacidad de satisfacción de sus necesidades y según unas políticas tecnológicas autóctonas enraizadas en el medio y en la sociedad. La correspondiente innovación tecnológica no puede estar basada en la transferencia tecnológica estricta, sino que ha 
de buscar y encontrar las tecnologías apropiadas que sirvan de instrumentos a los seres humanos sin afectar la naturaleza ni sus ecosistemas. Por otro lado, sus habitantes deben cuidar su entorno, ya que dependen de un medio ambiente sano que les provea de alimentos y recursos, agua potable y aire limpio. El paradigma de la sostenibilidad constituye pues un cambio importante frente a los paradigmas dominantes de desarrollo económico en el país y América Latina.

\section{Investigaciones recientes sobre sentidos y prácticas ambientales sostenibles en comunidades locales}

El programa DEL de la Unión Europea para Colombia se encuentra adelantando desde el 2011, un proyecto de buenas prácticas ambientales en el Resguardo Indígena Kankuamo, en las Poblaciones de Guatapurí, Chemesquemena, Atánquez y Los Haticos que busca aumentar la generación de ingresos a las comunidades indígenas mediante el aprovechamiento sostenible de la oferta florística y otros recursos de la Sierra Nevada de Santa Marta, denominado "ALTERNATIVA PILOTO PARA LA GENERACIÓN DE INGRESOS A COMUNIDADES INDIGENAS MEDIANTE EL APROVECHAMIENTO SOSTEBIBLE DE LOS PRODUCTOS NO MADERABLES DEL BOSQUE EN LA SIERRA NEVADA DE SANTA MARTA". En ese sentido, se implementan tecnologías para el apro- vechamiento sostenible de los recursos asociados al bosque, se brinda asistencia técnica y capacitación a productores indígenas en el uso de tecnologías para el aprovechamiento sostenible de los recursos asociados al bosque y se desarrolla una estrategia para la generación de valor a la producción y de mercadeo a la misma.

Valencia (2013) sostiene que en Antioquia, hay otro caso muy interesante de prácticas ambientales sostenibles, por parte de la Corporación para la Investigación y el Eco desarrollo Regional (CIER). Resulta que mediante la educación para el desarrollo rural, se buscan generar mayor sensibilidad en la sostenibilidad y el amor de las comunidades por su territorio y su medio ambiente. De esta manera, cincuenta y cinco tutores y diez empleados administrativos dirigen la educación de 1500 campesinos de Medellín, Sopetrán, Ebéjico, Buriticá, Caicedo, Betulia, Uramita, Caramanta, Peque y Olaya. Además de esto, en el corregimiento Llanadas, de Olaya, CIER tiene una finca de ganado doble propósito para necesidades familiares y cultivos como frijol y maíz. Allí mantienen semillas de comunidades rurales. Trabajan por la sostenibilidad y la seguridad alimentaria.

Incluso, en la misma ciudad de Barranquilla, podemos encontrar prácticas ambientales favorables a la Ciénaga de Mallorquín, que merece la pena destacar. El 22 de marzo del año 2014, el DAMAB celebró en la Ciénaga de Mallorquín el 
Día Mundial del Agua. Para ello realizó con estudiantes de Instituciones Educativas Distritales, un ciclo de sensibilización que denominó 'Cuido el Agua, cada gota es vida', en la que se alertaba sobre el cuidado, ahorro y uso eficiente del agua, en las instalaciones de las Aulas Ambientales, ubicadas en el Corregimiento de La Playa - Ciénaga de Mallorquín.

En este sitio participaron cerca de 250 participantes: estudiantes, animadores comunitarios, líderes cívicos, miembros de las Asociaciones de Pescadores, Policía Ambiental y directivos del Damab. El propósito de dicho evento fue sensibilizar a las comunidades y a los estudiantes del Distrito de Barranquilla, sobre la importancia de contribuir a diario con la preservación del único humedal que posee el Distrito: La Ciénaga de Mallorquín, todo esto en el marco de la celebración mundial del Día Mundial del Agua.

Para la directora del DAMAB, con esta actividad que se realizó en La Ciénaga de Mallorquín se busca,

Centrar todas las miradas e intenciones para que todas aquellas entidades del orden distrital y nacional den celeridad a los procesos de recuperación de este cuerpo de agua, en esta celebración debemos reflexionar sobre la importancia del cuidado de todos los recursos hídricos con los que contamos en nuestro entorno inmediato, hoy queremos unirnos a ese clamor mundial para cuidar ese preciado líquido, tan vital para nuestra existencia , y que escenario más apropiado que este para generar conciencia en la comunidad y las autoridades ambientales sobre el cuidado que debemos tener con el agua (Región Caribe, 2013).

Con respecto a este último punto, cabe destacar una iniciativa anterior que buscaba contribuir a la recuperación de la Ciénaga de Mallorquín Correa (2010), nos referimos a la solución parcial para tratar las aguas residuales en Barranquilla por parte del DAMAB, en la que se construyeron una serie de piscinas con el propósito de que el trabajo sucio de limpiar el agua se dejara a las bacterias para luego inyectar oxígeno. El destino final es el Arroyo León, que conduce a la Ciénaga. El proyecto de la planta de tratamiento, que tuvo un costo cercano a los \$18.000 millones y empezó a operar hace varios años, implicó para la entidad ambiental destinar entre el 50 y el $60 \%$ de su presupuesto a este sólo proyecto. Sin embargo, hasta la fecha, los resultados han sido mínimos, en parte porque no se ha encontrado solución a los 3.200 litros de agua residuales por segundo que producen los barranquilleros.

Por lo tanto, la enseñanza que dejan estas experiencias nos indica que la recuperación de la Ciénaga de Mallorquín pasa por varios frentes, que deben ser observados y tratados por las autoridades ambientales y los ciudadanos. En 
efecto, la Ciénaga cuenta con diversas problemáticas que no sólo tienen que ver con lo ambiental, sino también en lo social, lo económico y lo cultural. Así que las medidas o iniciativas que se adopten, deben ir encaminadas a solucionar de manera definitiva o por lo menos, en gran parte, los problemas de este humedal y su área de influencia. La concientización y sensibilización hacia los habitantes de esa zona, es un inicio muy importante para transformar la realidad de la Ciénaga de Mallorquín.

\section{Conclusiones}

El efecto acumulado del deterioro ambiental que se ha llevado a cabo durante los últimos cien años, se encuentra sintetizado en el desgaste de los ecosistemas en todo el mundo, desde los bosques y tundras hasta los humedales y sistemas costeros. Uno de los rasgos más preocupantes del desgaste ambiental es la reducción en la capacidad de los ecosistemas para prestar los servicios de aprovisionamiento que son tan vitales para el ser humano. En los últimos cincuenta años los seres humanos han transformado muchos ecosistemas del planeta más rápida e intensivamente que durante cualquier otro periodo comparable. En buena medida, esos cambios se hicieron para responder a la demanda creciente de alimentos, agua, madera y energía. Pero hoy día, los ecosistemas más importantes del planeta han perdido su capacidad de prestar los servicios de aprovisionamiento que son fundamentales para el ser humano.

Una gran contribución de los debates, declaraciones e informes sobre desarrollo sostenible y humano, como alternativa a los modelos de desarrollo capitalistas en América Latina, fue trasladar el debate en torno al medio ambiente de la discusión sobre problemas locales de contaminación hacia una reflexión más sistemática sobre las tasas de utilización y sobreexplotación de los recursos naturales en el mundo. Siguiendo a Nadal (2007) el enfoque tendrá que ser integral y sistémico. La problemática no es sólo técnico-ecológica, sino al mismo tiempo social y política. Estas son la calidad y la dimensión intrínseca del desarrollo sustentable El desarrollo sustentable habrá de resultar más bien del conjunto de las políticas públicas en lo económico, lo social, lo ambiental y aún lo cultural, y de la participación ciudadana que de programas aislados, incluso el ambiental en sí. O sea que, en las condiciones presentes, no puede afirmarse que la idea del desarrollo sustentable haya penetrado en la sociedad ni en los gobiernos al grado de que sea una base firme de formulación de políticas de desarrollo, donde éstas lleguen a considerarse necesarias. Dentro de cada nación, será indispensable que el desarrollo sustentable sea un objetivo colectivo, no limitado a algún sector o alguna zona crítica.

En lo concerniente a la Ciénaga de Mallorquín, hemos visto que las necesi- 
dades de recuperación no son sólo en el campo ambiental, sino también en el social y cultural. Para construir verdaderas soluciones a las problemáticas de este humedal, es necesario que las iniciativas que se piensen adoptar sean integrales con todos los frentes que afectan a la población. Precisamente, es a partir del protagonismo real de las personas, en que se puede lograr una verdadera transformación de la Ciénaga, ya que estos y sus respectivas actividades cumplen juegan un papel muy importante en la reconstrucción de dicho humedal. Es por tal razón, que la educación y la concientización de sus habitantes es un buen paso para cambiar las condiciones ambientales y culturales del sector.

Debe estimularse la generación de soluciones creativas que emanen desde abajo (las personas marginadas que padecen las consecuencias más graves de la contaminación de la Ciénaga) hacia arriba (autoridades nacionales y locales) y resulten, por lo tanto, más congruentes con las aspiraciones reales de las personas. Un Desarrollo a Escala Humana, tal como lo afirma Manfred MaxNeeef (1998), permitirá orientar en gran medida hacia la satisfacción de las necesidades humanas, exige un nuevo modo de interpretar la realidad. Nos obliga a ver y a evaluar el mundo, las personas y sus procesos, de una manera distinta a la convencional. Del mismo modo, la puesta en marcha de prácticas ambientales sostenibles en la Ciénaga de Mallorquín, debe apuntar hacia la satisfacción de las necesidades humanas y ambientales para un verdadero desarrollo integral.

\section{Referencias}

Baumol, W. (2004) The Free-Market Innovation Machine: Analyzing the Growth Miracle of Capitalism. Princenton, N. J.: Princenton University Press.

Brudtland, G. H. (1987). Informe Brudtland. Ginebra: ONU.

Cervantes, G. (2005) Desarrollo Sostenible. Univ. Politèc. de Catalunya.

CEPALC (2001). Una década de luces y sombras: América Latina y el Caribe en los años noventa. Bogotá: Naciones Unidaa/ CEPALC, en coedición con alfaomega.

Correa, P. (2010). Comienza a renacer la Ciénaga de Mallorquín. Bogotá, El Espectador.

Dos Santos, T. (2011). Imperialismo y dependencia. Caracas: Fundación Biblioteca Ayacucho.

Harlem, G (1984). Informe de la Comisión Mundial sobre el Medio Ambiente y el Desarrollo: "Nuestro Futuro Común". Ginebra, Organización de Naciones Unidas (ONU).

Mann, S. (2011). Sustainable Lens: A visual Guide. Washington, EEUU: CreateSpace. 
Max-Neef, M, Elizalde, A \& Hopehayn, M (1998). Desarrollo a escala humana. Montevideo: Editorial NormanComunidad.

Meadows, D (1972). Los Límites al crecimiento: informe al Club de Roma sobre el predicamento de la humanidad, Fondo de Cultura Económica.

Nadal, A. (2007). Obras escogidas de Víctor L. Urquidi. Desarrollo sustentable y cambio global. México D.F.: El colegio de México.

Organización de las Naciones Unidas para la Educación, la Ciencia y la Cultura (2012). Educación para el desarrollo sostenible. París: UNESCO.

PNUMA- ORPALC, GEO (2000). América Latina y el Caribe. Perspectivas del medio ambiente. México D.F. Oficina Regional para América Latina y el Caribe.

Programa de las Naciones Unidas para el desarrollo -PNUD- (1992). Desarrollo humano: Informe, Bogotá, Tercer Mundo.

Programa de las Naciones Unidas para el desarrollo -PNUD-. (2000). Informe sobre desarrollo humano. Madrid: Ediciones Mundi.

Región Caribe (2013). Celebran el día del agua en la Ciénaga de Mallorquín. Disponible en: http://regioncaribe. org/celebran-el-dia-del-agua-en-lacienaga-de-mallorquin/
Sarmiento, E. (1989). Los nuevos desafíos del desarrollo: fundamentos $y$ políticas. Bogotá: Tercer Mundo.

Silva, J. (2007). Crecimiento Económico y desarrollo humano. Bogotá: Academia Colombia de Ciencias Económicas.

Sarmiento, E. (2010). Desafíos del desarrollo. Bogotá: El Espectador.

UNEP- PNUMA (1999). Global Enviroment Outlook 2000., "Prólogo". Londres, UNEP/Earthscan

Unión Europea para Colombia (2011). ALTERNATIVA PILOTO PARA LA GENERACIÓN DE INGRESOS A COMUNIDADES INDIGENAS MEDIANTE EL APROVECHAMIENTO SOSTEBIBLE DE LOS PRODUCTOS NO MADERABLES DEL BOSQUE EN LA SIERRA NEVADA DE SANTA MARTA. Disponible en: http:// www.mipymes.gov.co/descargar. php?idFile=3695.

United Nations Environment Programme (UNEP-PNUMA, 1997). Global Environment Outlook (GEO-1). Executive summary: Global Overview.

Valencia, J (2013). Dan ejemplo con sus prácticas ambientales. Medellín, El Colombiano.

United Nations Environment Programme (UNEP-PNUMA, 1997). Global Enviroment Outlook 2000., "Prólogo". Londres, UNEP/Earthscan, pp. 12 y 13. 